\title{
CARACTERÍSTICAS FOLIARES Y TASAS VITALES DE ÁRBOLES SUCESIONALES TARDÍOS DE UN BOSQUE TROPICAL PERENNIFOLIO
}

\author{
Cristina Martínez-Garza ${ }^{1,3}$ y Henry F. Howe ${ }^{2}$ \\ ${ }^{1}$ Centro de Educación Ambiental en Biodiversidad y Conservación, Universidad Autónoma del Estado de Morelos \\ ${ }^{2}$ Department of Biological Sciences, University of Illinois at Chicago \\ ${ }^{3}$ Autor para la correspondencia: cristina.martinez@buzon.uaem.mx
}

\begin{abstract}
Resumen: Para acelerar la sucesión vegetal y restaurar la vegetación en áreas degradadas (e.g., pastizales abandonados) se ha sugerido utilizar especies sucesionales tardías. Para determinar cómo la dinámica foliar está relacionada con altas tasas de crecimiento y supervivencia en sitios sucesionales tempranos, evaluamos la hipótesis de que la plasticidad en la demografía foliar está positivamente correlacionada con el establecimiento de árboles de siete especies sucesionales tardías creciendo en ambientes soleados (pastizal y borde) y obscuros (bosque secundario) en Los Tuxtlas, Veracruz, México. Se monitorearon cohortes de hojas de 517 individuos de siete especies arbóreas creciendo en los tres hábitats durante un año. Los individuos que crecieron en bosque secundario y borde tuvieron mayor supervivencia foliar y menor producción de hojas que aquellos que crecieron en pastizal. Los individuos con mayor crecimiento y supervivencia en pastizal tuvieron una plasticidad alta en su supervivencia foliar y plasticidad baja en su producción de hojas (estabilidad), mientras que los individuos en el borde y el bosque con tasas altas de supervivencia fueron aquello que tuvieron estabilidad alta en su producción foliar. Nuestros resultados indican que Nectandra ambigens, Licaria velutina y Pouteria rhynchocarpa pueden ser sembradas en pastizales ya que muestran tasas altas de crecimiento y supervivencia en esos hábitats, además alcanzan diferentes estaturas a la edad adulta por lo que proveerían de diversidad estructural al bosque resultante. Especies con supervivencia baja en pastizales como Calophyllum brasiliense, Pimenta dioica, Amphitecna tuxtlensis y Eugenia inirebensis podrían sembrarse en grandes cantidades o hasta que un dosel de pioneras se haya formado.

Palabras claves: plantaciones experimentales, estabilidad fenotípica, árboles no-pioneros
\end{abstract}

\begin{abstract}
Restoration of vegetation in perturbed areas (e.g., abandoned pastures) with late-successional tree species may help to bypass decades of low diversity by accelerating succession. To elucidate how leaf dynamics is related to high growth rates and survival in early successional environments, we evaluated the hypothesis that plasticity in foliar demography is positively related to establishment of seven late-successional tree species growing in sunny sites (pasture and edge) and dark sites (secondary forest) at Los Tuxtlas, Veracruz, Mexico. We monitored leaves from 517 individuals from all species at all habitats during one year. Individuals growing in secondary forest and edge showed higher leaf survival and lower leaf production than those growing in pasture. Individuals with higher growth rates and survival in pasture showed high plasticity in their leaf survival and low plasticity in their leaf production (stability) while individuals in edge and secondary forest with high survivorship were those with high stability in their leaf production. Our results indicate that Nectandra ambigens, Licaria velutina and Pouteria rhynchocarpa may be planted in pastures because they show high growth rates and survival there, besides, they have different maximal height as adults and therefore they will provide structural diversity to the restored forest. Species with low plant survivorship in pastures as Calophyllum brasiliense, Pimenta dioica, Amphitecna tuxtlensis and Eugenia inirebensis should be planted in great numbers or until a canopy of pioneers develop.
\end{abstract}

Key words: synthetic communities, phenotypic stability, late-successional trees

I nvestigar cómo crecen las especies sucesionales tardías en sitios sucesionales tempranos resulta relevante por la alta deforestación en los trópicos que muchas veces es seguida por el abandono de las tierras y por procesos de regeneración natural (Moran et al., 1994). Es importante generar conocimiento aplicado al sembrar especies sucesionales tardías fuera del bosque, ya que el determinar su crecimiento ayudaría a predecir su respuesta al cambio climático global. En este contexto, las plantaciones experimentales pueden ser herramientas para entender los procesos ecológicos que dirigen el ensamblaje de las comunidades (Jordan et al., 1987).

El crecimiento de los árboles se ve afectado por múltiples factores entre los que se encuentran características de sus hojas como la longevidad, tasa de fotosíntesis, masa foliar 
por unidad de área y concentración de nitrógeno (Meavedel-Castillo, 1987; Lambers y Poorter, 1992). Estas características cambian de forma simultánea y predecible en respuesta a la calidad de la luz, la concentración de nutrientes y la disponibilidad de agua (Reich et al., 1992). Reemplazar una hoja o producir hojas nuevas es costoso, por lo que la retención de hojas es adaptativa (Chabot y Hicks, 1982). Cuando se abre un claro en el dosel o durante la estación seca, aumentan los recursos lumínicos provocando que las tasas de pérdida y producción de hojas aumenten (Bongers y Popma, 1990; Reich et al., 2004). Bajo condiciones de máxima luminosidad, las plantas pierden sus hojas si se presenta estrés hídrico y desarrollan hojas nuevas sólo si hay disponibilidad de agua (Carabias-Lillo y Guevara-Sada, 1985; ver Reich et al., 2003). En resumen, el recambio de hojas es alto en condiciones de alta luminosidad y es bajo en el sotobosque, lo que tiene efectos directos en el crecimiento y la supervivencia de las plantas.

La tasa de producción y mortalidad de hojas se conoce como demografía foliar y difiere entre especies con diferente historia de vida. En los trópicos, los árboles sucesionales tempranos (pioneros) tienen longevidad foliar corta y alta producción de hojas en comparación con los árboles sucesionales tardíos (Coley, 1988). Esta demografía foliar refleja la adaptación de las especies pioneras para crecer rápidamente evitando condiciones de estrés hídrico (Reich et al., 1991). Estas especies pueden germinar en el sotobosque pero sus plántulas son incapaces de mantener su área foliar debido a la falta de luz que limita el recambio de sus hojas; bajo las mismas condiciones, plántulas de árboles sucesionales tardíos se mantienen vivas reteniendo sus hojas y produciendo escasas hojas nuevas (King, 1994). Sin embargo, las especies sucesionales tardías que sobreviven en la sombra del sotobosque muestran una alta variación en su longevidad y en su producción foliar. Para juveniles de 7 especies en Panamá (King, 1994) y de 25 especies en Bolivia (Poorter y Bongers, 2006) se encontró una variación en la longevidad foliar de 4 y 5 veces respectivamente; para adultos de 16 especies en Malasia, esta variación fue de 13 veces (Osada et al., 2001). La variación en la producción foliar de los juveniles y adultos fue de 1.5 y 3 veces en Panamá y Malasia respectivamente. Los árboles pioneros dominan los ambientes sucesionales tempranos pero se ha descubierto que los árboles tardíos pueden crecer bien si son sembrados en zonas abiertas (Martínez-Garza y Howe, 2003). Se ha discutido que esta capacidad se relaciona a su adaptación al gradiente vertical y horizontal de luz que existe dentro del bosque tropical. Estos árboles desarrollan hojas grandes y delgadas en la sombra y hojas gruesas y pequeñas en condiciones de luz (Popma et al., 1992) lo que les permite utilizar eficientemente la escasa luz del sotobosque o la alta luminosidad en la parte alta del dosel. Esta plasticidad fenotípica en las características foliares de los árboles del bosque está asociada a la disponibilidad inmediata de re- cursos además de tener un componente genético (Bradshaw, 1965). En bosques tropicales de Malasia se encontró que los árboles dominantes del dosel muestran plasticidad en la tasa de recambio foliar dentro de su dosel: tasas altas en el dosel superior y tasas de recambio bajas en su dosel inferior (Osada et al., 2001). Mientras que en bosques de la región de Los Tuxtlas, Veracruz, se encontró que una plasticidad alta en la masa foliar por unidad de área (peso foliar especifico, PFE, $\mathrm{g} \mathrm{m}^{-2}$ ) contribuye a tasas altas de crecimiento y supervivencia de juveniles de especies sucesionales tardías creciendo en sitios bajo sucesión temprana (Martínez-Garza et al., 2005). Sin embargo, es de esperarse que especies que evolucionaron bajo condiciones ambientales permanentemente desfavorables, como el sotobosque del bosque perennifolio, muestren una plasticidad baja en algunas características (Lortie y Aarssen,1996). Por ejemplo, en un bosque húmedo de Panamá se encontró que los arbustos pequeños de Psychotria que habitan el sotobosque, no responden al aumento de recursos cuando se abren claros en el dosel (Valladares $e t$ al., 2000), lo que corresponde a una respuesta poco plástica de una especie adaptada a un ambiente estable (estabilidad fenotípica). Aquí se presenta un estudio de un año donde se evaluó si la plasticidad en la demografía foliar de juveniles de siete árboles sucesionales tardíos está asociada a su crecimiento y supervivencia en sitios sucesionales tempranos (pastizal y borde) y en un bosque secundario viejo. Además de la plasticidad en la morfología, nuestra pregunta principal fue si los patrones de plasticidad en la demografía foliar de árboles juveniles sucesionales tardíos de diferentes estaturas pueden pre-adaptarlos o interferir negativamente en su crecimiento y supervivencia en sitios donde no evolucionaron (sucesionales tempranos). Se esperaba que las especies que respondieran inmediatamente a la disponibilidad de recursos con tasas altas de recambio foliar en pastizales y tasas bajas de recambio en el bosque (plasticidad alta), sobrevivieran y crecieran mejor en todos los hábitats. Aquellas especies sucesionales tardías con tasas de crecimiento y supervivencia mayores en pastizales serán ideales para proyectos de restauración, donde se busca no sólo recuperar la vegetación, sino restaurar la biodiversidad característica del bosque primario. Las especies sucesionales tardías que muestren plasticidad alta en su demografía foliar, seguirán mostrando tasas altas de crecimiento y supervivencia bajo sombra, cuando sean rebasadas por árboles de crecimiento más rápido, lo que les permitirá permanecer en el bosque primario.

\section{Materiales y Métodos}

Sitio de estudio. El estudio se llevó a cabo en una plantación experimental en el Ejido de Lázaro Cárdenas cerca de la Estación de Biología Tropical "Los Tuxtlas" (EBTLT) en Veracruz, México (18³0' N y 9503' W). La EBTLT se encuentra dentro de una Reserva de 640 ha de bosque tropical 
perennifolio que corresponde al limite norte de este tipo de bosque para el continente. El Ejido de Lázaro Cárdenas está localizado en la esquina suroeste de la Reserva. El suelo en esta zona es arenoso, clasificado como andosol (MartinDel-Pozzo, 1997). La temperatura media anual es de $27^{\circ} \mathrm{C}$ y la precipitación es de $4900 \mathrm{~mm}$ al año. Existe una pequeña estación seca de marzo a mayo, y la época de lluvias se extiende de junio a febrero; la precipitación en el año de estudio estuvo dentro de la variación reportada para los últimos 10 años (R. Coates, com. pers.). En la Reserva, el bosque primario tiene un dosel cerrado de $35 \mathrm{~m}$ de altura. Nectandra ambigens (Blake) C.K. Allen (Lauraceae) es el árbol más común en el dosel y Pseudolmedia oxyphyllaria Donn. Sm. (Moraceae) y Astrocaryum mexicanum Liebm. Ex Mart (Arecaceae) son los más abundantes en el dosel medio y en el sotobosque, respectivamente (Bongers et al., 1988). La reserva está rodeada por campos de cultivo, pastizales y fragmentos de bosque de diferentes tamaños.

La plantación experimental está localizada dentro de una parcela de 25 ha que fue deforestada en 1968, cultivada por 4 años y utilizada como pastizal por los siguientes 15 años. Esta área ha estado bajo regeneración natural desde que fue abandonada en 1987. El bosque secundario tiene un dosel de $15 \mathrm{~m}$ de altura con especies pioneras como Cecropia obtusifolia Bertol. (Moraceae), Heliocarpus appendiculatus Turcz. (Tiliaceae), Bursera simaruba Sarg. (Burseraceae) y Cedrela odorata L. (Meliaceae); mientras que el ambiente sucesional temprano está compuesto de dos microhábitats bien diferenciados: (1) pastizal abierto de aproximadamente 3 ha cubierto con el pasto exótico Cynodon plectostachyum (K. Schum.) Pilg. que llega a medir hasta $1.5 \mathrm{~m}$ de altura durante la época de lluvias. El pastizal tiene, además, algunos árboles aislados de especies pioneras; y (2) un bosque joven con un dosel discontinuo de 2 a $4 \mathrm{~m}$ de altura constituido con las mismas especies pioneras que se encuentran en el pastizal y en el bosque secundario aledaño; a este microhábitat lo llamaremos borde.

La plantación experimental fue establecida en 1997 con 45 especies de palmas, lianas, árboles pioneros y sucesionales tardíos. Semillas de todas las especies fueron colectadas en la EBTLT de agosto a diciembre de 1996, y germinadas en un vivero bajo la sombra. Se obtuvieron en promedio 100 individuos por especie para un total de 5000 individuos en toda la plantación. Las semillas fueron colectadas de más de tres individuos para asegurar la diversidad genética. En marzo del 1997 se marcaron 5000 cuadros de $2 \times 2 \mathrm{~m}$ en toda el área que incluían pastizal, borde y selva secundaria aledaña. Las plántulas de las 45 especies mezcladas fueron sembradas en la cuadricula a los 4 meses de edad aproximadamente, con una plántula colocada en el centro de cada cuadro (Martínez-Garza et al., 2005). De la plantación experimental se seleccionaron siete árboles sucesionales tardíos de cinco familias que representan el intervalo de altura máxima de los árboles en este bosque: Amphitecna tuxtlensis (Bignonaceae, 3-10 m de altura máxima) y Pouteria rhynchocarpa (Sapotaceae, 3-8 m) son árboles del sotobosque, Eugenia inirebensis (Myrtaceae, 8-15 m) y Licaria velutina (Lauraceae, 10-15 m) crecen en el dosel medio y Calophyllum brasiliense (Clusiaceae, 20-30 m), Nectandra ambigens (Lauraceae, 20-40 m) y Pimenta dioica (Myrtaceae, $8-30 \mathrm{~m}$ ) son árboles del dosel o emergentes. En marzo del 2001, al comienzo de este estudio, los individuos seleccionados tenían 4 años de edad, al menos cinco hojas, entre 47 y $141 \mathrm{~cm}$ de altura y de 5.5 a $15 \mathrm{~mm}$ de diámetro en la base del tallo (Martínez-Garza et al., 2005). Se calcularon incrementos mensuales en altura (altura -altura $_{\mathrm{t} 1} /$ tiempo) y en diámetro en la base del tallo para tres periodos de tiempo entre cuatro censos; aquí se reporta el promedio de los incrementos mensuales. El porcentaje de supervivencia fue calculado como el número de individuos que sobrevivo de 1997 a 2001, dividido por el número de individuos presentes en 1997 para cada especie y cada hábitat. Se marcaron de 5 a 52 individuos por especie por hábitat y en cada individuo se marcaron hasta cinco hojas totalmente desarrolladas. Sólo se tomaron hojas colocadas al final de las ramas para tener hojas de edad comparable. La pérdida de las hojas marcadas y las hojas nuevas totalmente desarrolladas en una rama fueron censadas y marcadas después de 1, 6 y 12 meses. El censo de Abril del 2001 (después de un mes) coincidió con el periodo pico de producción foliar para las especies arbóreas de Los Tuxtlas (Carabias-Lillo y Guevara-Sada, 1985). En total, 2547 hojas de 517 individuos fueron marcadas y observadas para evaluar supervivencia foliar. El manejo de la plantación incluyó el corte de pastos y hierbas $50 \mathrm{~cm}$ alrededor de los individuos antes de cada censo y las plantas no fueron regadas ni fumigadas durante el experimento.

A cada individuo dentro de los tres hábitats se le asigno un índice de iluminación del dosel para caracterizar su microhábitat (Clark y Clark, 1992). Este índice es una medida cualitativa utilizada para caracterizar rápida y frecuentemente microhábitats variables de muchos individuos y va de 1 (sin luz directa sobre los individuos) hasta 5 (individuos totalmente expuestos a la luz). Los individuos con índices de 1 y 2 fueron clasificados como habitando el bosque secundario, aquellos individuos con índices de 3 y 4 el borde y aquellos con nivel 5 el pastizal. A los individuos bajo árboles aislados en medio del pastizal les correspondió el índice de iluminación del dosel de 3 por lo que se consideraron como habitando el borde; asimismo un individuo creciendo en un claro dentro del bosque secundario le correspondía un índice de 4 por lo que se le asignó el hábitat borde. Se midieron variables abióticas cerca de una submuestra de individuos en cada hábitat durante la estación seca del 2001: se midió la densidad instantánea de flujo de fotones con un medidor de Luz (Li-cor LI-189 quantum sensor), al medio día arriba del dosel de una muestra de los juveniles $(1.5 \mathrm{~m}, \mathrm{~N}=406)$ en los diferentes hábitats. Los individuos experimentaron diferencias significativamente en la densidad instantánea de flu- 
Cuadro 1. Densidad de flujo de fotones (DFF, mediana [max, min], $\mathrm{N}=406$ ), humedad del suelo y compactación (promedio ( $\mathrm{EE}, \mathrm{N}=135$ ) en tres hábitats de una plantación experimental en Los Tuxtlas, Veracruz, México.

\begin{tabular}{lccc}
\hline Hábitat & $\begin{array}{c}\text { DFF } \\
\left(\mu \mathrm{mol} \mathrm{m}^{-2} \mathrm{~s}^{-1}\right)\end{array}$ & $\begin{array}{c}\text { Humedad del suelo } \\
\left(\mathrm{ml} \mathrm{g}^{-1}\right)\end{array}$ & $\begin{array}{c}\text { Compactación } \\
\left(\mathrm{g} \mathrm{cm}^{-3}\right)\end{array}$ \\
\hline Pastizal & $100(13,2227) \mathrm{c}^{1}$ & $0.42 \pm 0.01 \mathrm{~b}$ & $0.668 \pm 0.01 \mathrm{a}$ \\
Bordes & $33(9,1352) \mathrm{b}$ & $0.43 \pm 0.01 \mathrm{a}$ & $0.667 \pm 0.01 \mathrm{a}$ \\
Bosque & $23(9,163) \mathrm{a}$ & $0.45 \pm 0.01 \mathrm{a}$ & $0.666 \pm 0.01 \mathrm{a}$ \\
\hline
\end{tabular}

${ }_{1}^{1}$ Las letras después de las medias o promedios indican diferencias significativas $(\mathrm{P}<0.05)$.

jo de fotones en los diferentes hábitats (Kruskall-Wallis ${ }_{(2,402)}=$ 54.93, $\mathrm{P}<0.0001$; Cuadro 1). Se tomaron 135 muestras de suelo a $20 \mathrm{~cm}$ de los individuos y a una profundidad de $5 \mathrm{~cm}$ durante la mañana. Con estas muestras se evaluó el contenido de humedad y la densidad del suelo, está última como un estimador de la compactación del suelo (Chapman, 1976). El pastizal mostró los suelos más secos $\left(\mathrm{F}_{(2,131)}=4.65, \mathrm{P}<\right.$ 0.01) mientras que la densidad del suelo fue similar para los tres hábitats $\left(\mathrm{F}_{(2,131)}=0.01, \mathrm{P}>0.5\right)$. Las condiciones microambientales en el bosque secundario de 10 años fueron similares a las encontradas en un bosque primario (ver por ejemplo Holl, 1999), por lo que sería el ambiente natural de establecimiento de las especies sucesionales tardías (de aquí en adelante llamado bosque). El índice de iluminación del dosel mostró que menos del $30 \%$ de los juveniles experimentó un descenso anual en los niveles de luz debido al proceso de sucesión natural (datos no publicados).

Análisis de Datos. La supervivencia de las hojas (1x foliar) se reporta como la proporción promedio de hojas vivas de cada individuo al final de cada periodo (1, 6 y 12 meses). Para probar si la supervivencia de las hojas de las siete especies era diferente en los tres hábitats, se hizo un ANOVA de medidas repetidas. En este análisis el logaritmo natural de la supervivencia de las hojas (In 1x foliar; Begon et al., 1987) fue la variable dependiente y el tiempo fue el factor repetido (tres niveles). La producción foliar se midió como la suma de las hojas producidas por individuo después de los tres censos. Los datos de producción foliar no se pudieron normalizar por lo que se hicieron análisis no-paramétricos de Kruskall-Wallis: uno con el hábitat como factor independiente con tres niveles y el otro con las especies como factor independiente con siete niveles, en ambos la producción foliar como variable dependiente; las pruebas significativas se analizaron con comparaciones múltiples de rangos medios (Siegel y Castellan, 2005). La plasticidad en la supervivencia de las hojas se midió como el coeficiente de variación de la supervivencia de las hojas de los individuos en los tres hábitats: $\mathrm{CV}=[($ desviación estándar de $\ln 1 \mathrm{x}$ foliar $\times$ 100)/ (promedio ln lx foliar)]; la plasticidad en la producción de hojas en los tres habitats se calculó de manera similar (Schlichting y Levin, 1984). Esta medida de plasticidad permite la comparación de la variación intraespecifica de la demografía foliar de siete especies creciendo en pastizal, borde y bosque. Para probar que variables podrían explicar las tasas de crecimiento y supervivencia de las especies en los diferentes hábitats se hicieron correlaciones de Pearson de los incrementos mensuales en altura $\left(\mathrm{cm} \mathrm{mes}^{-1}\right)$, diámetro $\left(\mathrm{mm} \mathrm{mes}^{-1}\right)$ y la supervivencia de juveniles (\%) con el promedio de la supervivencia foliar (ln lx foliar), la mediana de la producción foliar y la plasticidad en la demografía foliar. Los valores $\mathrm{P}$ de las correlaciones fueron ajustados para controlar por comparaciones múltiples (StatSoft, 2004). En los resultados se presenta promedio y error estándar o mediana con máximo y mínimo para los datos analizados con pruebas paramétricas o no-paramétricas, respectivamente. Para abreviar el texto se hace referencia sólo al género de las especies estudiadas. Todos los análisis estadísticos fueron realizados en STATISTICA 7.0 (StatSoft, 2004).

Cuadro 2. Especies de árboles del dosel ( $>30 \mathrm{~m}$ altura), dosel medio ( $>10 \mathrm{~m}$ altura) y sotobosque $(<10 \mathrm{~m}$ altura), promedio anual de supervivencia foliar \pm EE (proporción de hojas / año), mediana anual de producción foliar (max, min) (número de hojas /año) y plasticidad en ln de supervivencia foliar (\%) y en la producción foliar (\%) de siete especies sucesionales tardías en Los Tuxtlas, Veracruz, México.

\begin{tabular}{|c|c|c|c|c|c|c|}
\hline \multirow[t]{2}{*}{ Especies $^{1}$} & \multirow[t]{2}{*}{ Familia } & \multirow[t]{2}{*}{ Estrato } & \multirow{2}{*}{$\frac{\text { Promedio }}{\text { Supervivencia }}$} & \multirow{2}{*}{$\begin{array}{c}\text { Mediana } \\
\text { Producción }\end{array}$} & \multicolumn{2}{|c|}{ Plasticidad } \\
\hline & & & & & In supervivencia & Producción \\
\hline Amphitecna tuxtlensis A.H. Gentry & Bignonaceae & Sotobosque & $0.85 \pm 0.03 \mathrm{ab}^{2}$ & $8(0,27) \mathrm{a}$ & -16.59 & 5.62 \\
\hline Calophyllum brasiliense Cambess. & Clusiaceae & Dosel & $0.92 \pm 0.03 \mathrm{a}$ & $4.5(0,14) a b$ & -37.57 & 13.13 \\
\hline Eugenia inirebensis P.E. Sánchez & Myrtaceae & Dosel medio & $0.89 \pm 0.03 \mathrm{ab}$ & $7(2,12) a b$ & -43.69 & 13.93 \\
\hline Licaria velutina van der Werff & Lauraceae & Dosel medio & $0.81 \pm 0.01 \mathrm{ab}$ & $10(0,27) \mathrm{a}$ & -11.52 & 3.97 \\
\hline Nectandra ambigens (S.F. Blake) C.K. Allen & Lauraceae & Dosel & $0.81 \pm 0.02 b$ & $4(0,15) a b$ & -13.80 & 9.70 \\
\hline Pimenta dioica (L.) Merr. & Myrtaceae & Dosel & $0.85 \pm 0.03 \mathrm{ab}$ & $8(2,17) a b$ & -28.39 & 9.21 \\
\hline Pouteria rhynchocarpa T.D. Penn. & Sapotaceae & Sotobosque & $0.90 \pm 0.02 \mathrm{a}$ & $4(0,12) b$ & -27.16 & 10.68 \\
\hline
\end{tabular}

${ }^{1}$ Ibarra-Manriquez y Sinaca 1995, Ibarra-Manriquez y Sinaca 1996a, Ibarra-Manriquez y Sinaca 1996b

${ }^{2}$ Letras diferentes después de promedios o medianas indican diferencias significativas $(P<0.05)$. 
Cuadro 3. Correlaciones de Pearson $(r)$ de supervivencia de juveniles $(\%)$, incremento en altura $\left(\mathrm{cm} \mathrm{mes}^{-1}\right)$ e incremento en diámetro $\left(\mathrm{mm}^{\mathrm{m}} \mathrm{m} \mathrm{s}^{-1}\right)$ con el promedio del In de supervivencia foliar, plasticidad en In de supervivencia foliar (CV Supe Foliar, \%), producción foliar y plasticidad en la producción foliar (CV Prod Foliar, \%) de siete especies sucesionales tardías en Los Tuxtlas, Veracruz, México.

\begin{tabular}{lcccc}
\hline & & \multicolumn{2}{c}{ Características Foliares } \\
\cline { 2 - 5 } & Supervivencia Foliar & CV Sobr Foliar & Producción Foliar & CV Prod Foliar \\
\hline Supervivencia de las plantas en el bosque & -0.16 & $\mathbf{0 . 7 8}$ & 0.10 & $\mathbf{- 0 . 7 6}$ \\
Borde & -0.71 & $\mathbf{0 . 8 6}$ & 0.27 & $\mathbf{- 0 . 8 4}$ \\
Pastizal & $\mathbf{- 0 . 8 5}$ & $\mathbf{0 . 7 5}$ & 0.17 & -0.51 \\
\hline Incremento en altura en el bosque & -0.31 & 0.60 & 0.29 & -0.65 \\
Borde & -0.09 & 0.29 & 0.49 & -0.24 \\
Pastizal & -0.73 & -0.92 & -0.21 & -0.79 \\
\hline Incremento en diámetro en el bosque & 0.33 & 0.33 & 0.03 & -0.47 \\
Borde & $\mathbf{- 0 . 7 6}$ & $\mathbf{0 . 8 7}$ & 0.12 & -0.63 \\
Pastizal & -0.59 & & \\
\hline
\end{tabular}

${ }^{1}$ Correlaciones con $\mathrm{P}<0.05$ se muestran con negritas, $\mathrm{P}<0.005$ en negritas e itálicas

\section{Resultados}

Supervivencia Foliar. Tomando en cuenta las siete especies y los tres hábitats, la supervivencia foliar promedio fue de $0.86 \pm 0.02$ y varió significativamente entre las especies (Cuadro 2; $\mathrm{F}_{(6,440)}=2.94, \mathrm{P}<0.005$ ). Calophyllum, un árbol del dosel y Licaria, un árbol del dosel medio, tuvieron la supervivencia foliar más alta y más baja respectivamente. En general para todas las especies, la supervivencia foliar varió significativamente entre los hábitats $\left(\mathrm{F}_{(1,440)}=4.25, \mathrm{P}\right.$ $<0.01$ ); la supervivencia foliar fue significativamente más baja en el pastizal $(0.79 \pm 0.02)$ comparado con el bosque $(0.90 \pm 0.02)$ y el borde $(0.89 \pm 0.01)$, los cuales fueron estadísticamente iguales. La interacción de especie con hábitat no fue significativa $\left(\mathrm{F}_{(12,440)}=0.95, \mathrm{P}>0.5\right)$. La interacción significativa de tiempo con especie $\left(\mathrm{F}_{(6,440)}=2.73, \mathrm{P}<0.01\right)$ reveló que las especies mostraron diferencias significativas en su supervivencia foliar después de sólo 6 meses. La interacción significativa de tiempo con hábitat $\left(\mathrm{F}_{(4,880)}=4.26\right.$, $\mathrm{P}<0.001)$ mostró que la sobrevivecia foliar fue significativamente más baja para las plantas creciendo en el pastizal hasta después de 12 meses $\left(\mathrm{F}_{(6,440)}=5.51, \mathrm{P}<0.004\right)$.

Producción foliar. En total, 3592 hojas fueron marcadas como producidas en este periodo de tiempo. Tomando en cuenta las siete especies en los tres hábitats, la mediana anual de producción foliar fue de 5.8 hojas por individuo (mínimo 0, máximo 27). La producción foliar difirió entre las especies (Kruskall Wallis $\mathrm{H}_{(6,473)}=123.47 \mathrm{P}<0.0001$ ), y entre los hábitats (Kruskall Wallis $\mathrm{H}_{(2,469)}=7.96, \mathrm{P}<0.02$ ). Las comparaciones múltiples entre especies mostraron un gradiente en el que Licaria y Eugenia tuvieron la producción foliar más alta, estadísticamente similar a la de Amphitecna, un árbol del sotobosque y a Pimenta, un árbol del dosel. El análisis sugiere que Pouteria tuvo la producción foliar más baja, estadísticamente igual a la de los árboles del dosel Nectandra y Calophyllum. Las comparaciones múltiples entre hábitats mostraron que la producción foliar fue significativamente más alta para los individuos creciendo en el pastizal $[8(0,27)]$ comparada con la producción de los individuos creciendo en el bosque $[5(0,27)]$ y en el borde $[6(0,26)]$, la producción foliar fue estadísticamente igual en estos dos últimos hábitats.

Plasticidad. La plasticidad promedio, en valores absolutos fue casi tres veces mayor para la supervivencia de las hojas (25.53 $\pm 4.6 \%)$ que para la producción foliar $(9.46 \pm 1.4 \%)$. Licaria mostró la plasticidad más alta en supervivencia foliar (-11.51\%) y la plasticidad más baja en producción foliar (3.97 \%), mientras que Eugenia mostró el patrón opuesto: la plasticidad más baja en supervivencia foliar (-43.69\%) y la plasticidad más alta en producción foliar (13.93\%) (Cuadro 2).

Crecimiento, supervivencia y demografía foliar. La supervivencia y el crecimiento de las especies estudiadas estuvieron correlacionados con los promedios y la plasticidad en la demografía foliar en los tres hábitats (Cuadro 3). La supervivencia de los individuos creciendo en el pastizal estuvo negativamente correlacionada con el logaritmo de la supervivencia foliar promedio en ese hábitat (Figura 1, Cuadro 3) mientras que la plasticidad en esta variable estuvo positivamente correlacionada con la alta supervivencia de los individuos creciendo en el pastizal, borde y bosque (Figura 2, Cuadro 3,). En el bosque, esta correlación se debió principalmente a dos especies (Calophyllum y Eugenia) que mostraron la supervivencia más baja, mientras que en los otros dos ambientes la relación es continua.

El incremento en altura de las siete especies estuvo altamente correlacionado con la plasticidad en la supervivencia foliar de los individuos creciendo en el pastizal (Figura 3, Cuadro 3). El incremento promedio en diámetro para los individuos creciendo en el borde estuvo negativamente co- 


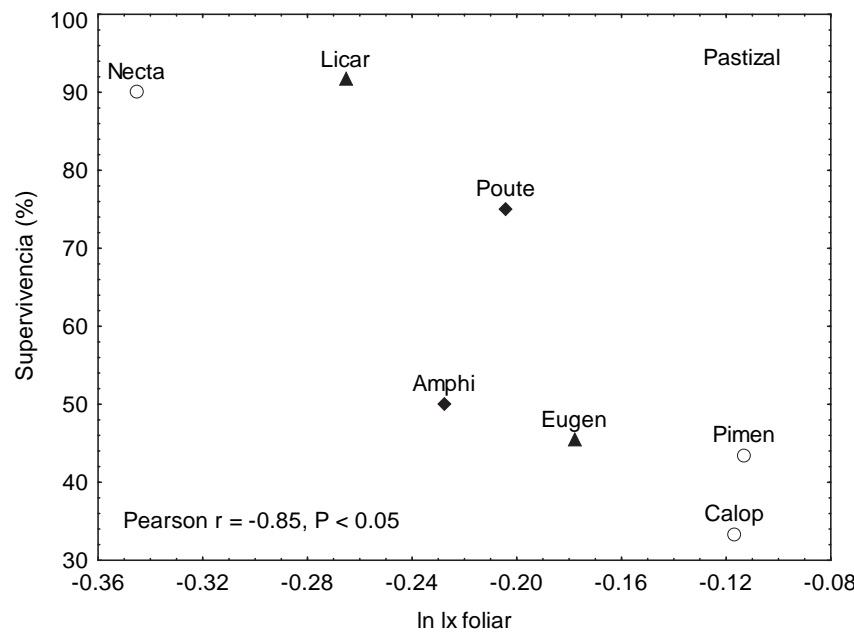

Figura 1. Correlación de la supervivencia de los individuos (\%) y el promedio del logaritmo natural de la supervivencia foliar (ln $1 \mathrm{x}$ foliar) de siete especies sucesionales tardías creciendo en pastizal de Los Tuxtlas, Veracruz, Mexico. Los círculos corresponden a árboles del dosel, los triángulos al dosel medio y los diamantes a los árboles que habitan el sotobosque. Se muestran las primeras cinco letras del género para cada especie.

rrelacionado con la supervivencia foliar promedio en ese hábitat (Cuadro 3); sin embargo, había un marcado efecto de Amphitecna, un árbol del sotobosque con supervivencia foliar muy baja e incremento en diámetro muy bajo; si se eliminaba esta especie, la correlación no era significativa $(\mathrm{r}=-0.51, \mathrm{P}<0.5, \mathrm{~N}=6)$. En el pastizal, el incremento en diámetro estuvo positivamente correlacionado con la plasticidad en la supervivencia foliar (Cuadro 3) con un efecto mayor de Eugenia, un árbol del dosel medio que mostró una plasticidad muy baja en la supervivencia foliar junto con un incremento en diámetro bajo; sin embargo, en este caso la correlación sigue siendo significativa si se elimina esta especie $(r=0.82, \mathrm{P}<0.05, \mathrm{~N}=6)$.

La supervivencia de los individuos y los incrementos en altura estuvieron correlacionados con la plasticidad en la producción foliar pero no con los valores promedio (Cuadro 3). La supervivencia de los juveniles en el bosque y el borde estuvo negativamente correlacionada con la plasticidad en la producción foliar (Figura 4, Cuadro 3). El incremento en altura de los individuos creciendo en el pastizal estuvo negativamente correlacionado con la plasticidad en la producción foliar (Cuadro 3).

\section{Discusión}

La restauración ecológica tiene diferentes objetivos, como evitar la erosión, reestablecer la fertilidad o recuperar la biodiversidad (Allen et al., 1997; Holl y Howarth, 2000). Tasas altas de crecimiento y supervivencia son atributos
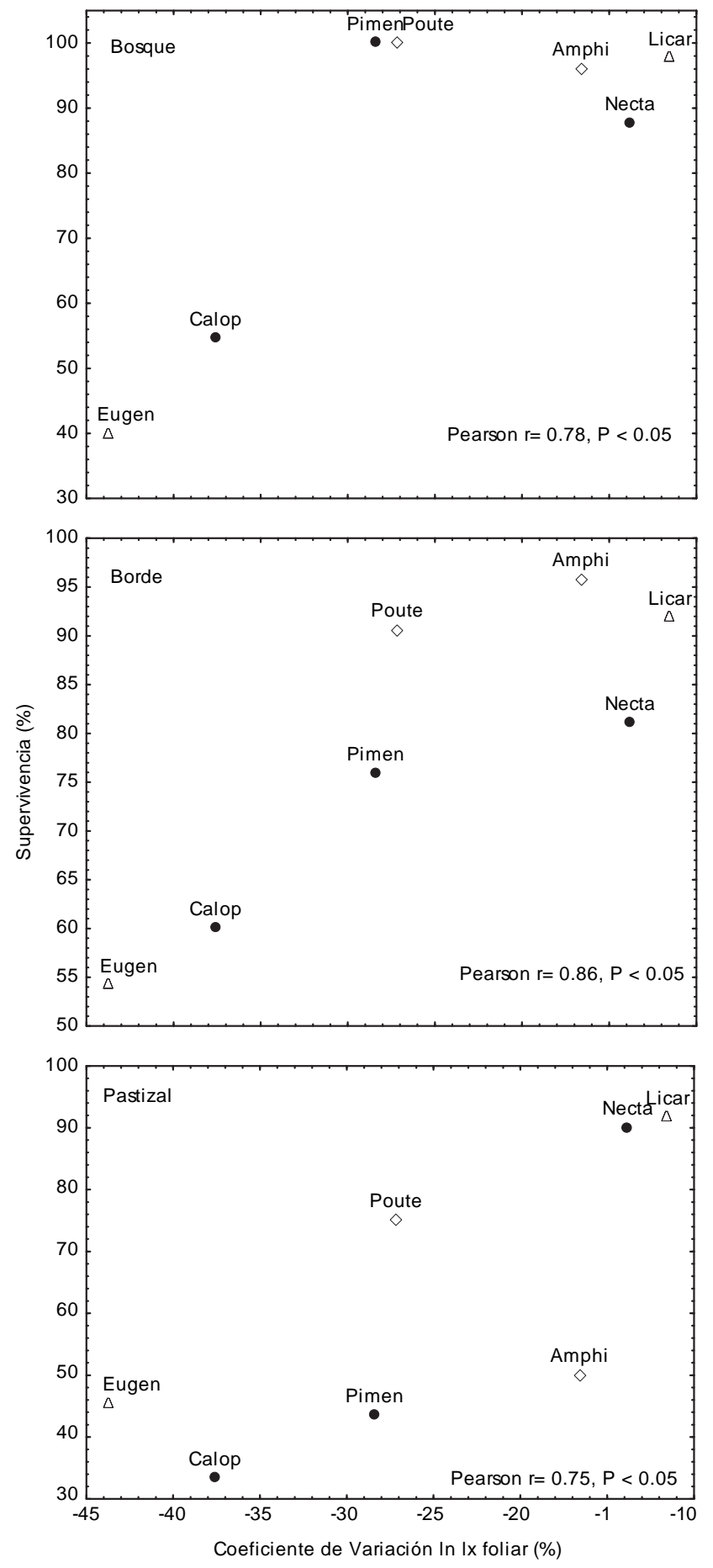

Figura 2. Correlación de la supervivencia de los individuos (\%)y la plasticidad de la supervivencia foliar (Coeficiente de Variación ln lx foliar; \%) de siete especies sucesionales tardías en tres hábitats de Los Tuxtlas, Veracruz, Mexico. Los círculos corresponden a árboles del dosel, los triángulos al dosel medio y los diamantes a los árboles que habitan el sotobosque. Se muestran las primeras cinco letras del género para cada especie. 


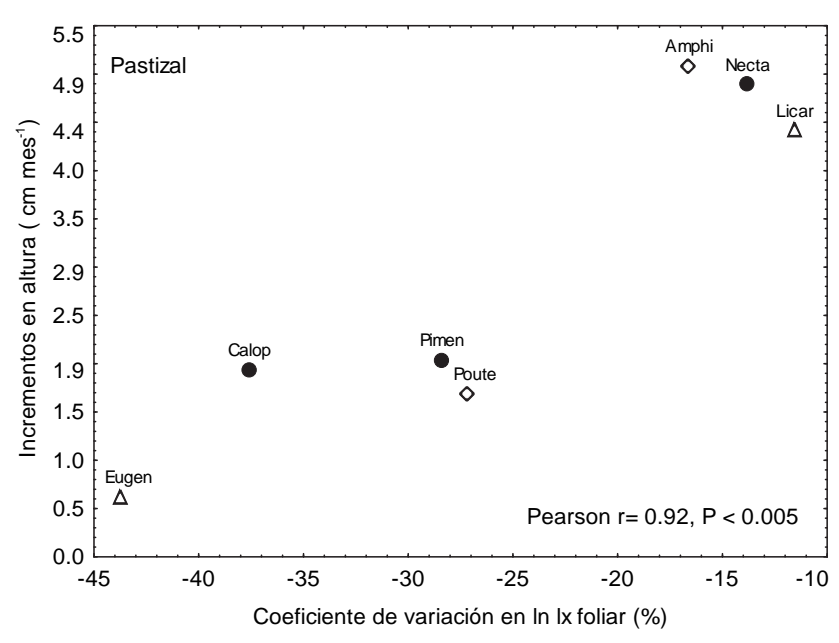

Figura 3. Correlaciones del incremento en altura $\left(\mathrm{cm} \mathrm{mes}^{-1}\right)$ y la plasticidad de la supervivencia foliar (Coeficiente de Variación ln lx foliar, \%) de siete especies sucesionales tardías creciendo en pastizales de Los Tuxtlas, Veracruz, Mexico. Los círculos corresponden a árboles del dosel, los triángulos al dosel medio y los diamantes a los árboles que habitan el sotobosque. Se muestran las primeras cinco letras del género para cada especie.

deseados en las especies que se utilizan para restaurar los ecosistemas. Sin embargo, las especies con tasas altas de crecimiento (pioneras), tienen supervivencia baja y representan menos del $20 \%$ de la diversidad de las leñosas del bosque tropical (Martínez-Garza y Howe, 2003). Las semillas de especies sucesionales tardías tardan mucho en llegar a sitios perturbados o bajo regeneración porque los animales que las dispersan no cruzan los pastizales (Martínez-Garza y González-Montagut, 1999; Wijdeven y Kuzee, 2000; Zimmerman et al., 2000). Estas especies se siembran en condiciones de alta luminosidad en proyectos de restauración ecológica o se ven expuestas a esas condiciones después de eventos masivos de deforestación (Martínez-Garza y Howe, 2003; Benitez-Malvido et al., 2005). Sin embargo, no se ha estudiando a detalle si estos árboles pueden crecer y sobrevivir en áreas perturbadas ya sea porque pueden cambiar su demografía foliar (plasticidad) en respuesta a la abertura de claros o porque mantienen su producción y longevidad foliar en el mismo nivel que en el sotobosque (estabilidad). Para las siete especies sucesionales tardías que estudiamos, la caída y producción de las hojas fue más rápida en el pastizal que en el sotobosque, como se espera si las especies responden inmediatamente a la disponibilidad de recursos (Martínez-Garza y Howe, 2005). Además, en el pastizal, aquellas especies con tasas altas de crecimiento y supervivencia mostraron plasticidad alta en su supervivencia foliar, como se esperaba, y plasticidad baja (estabilidad) en su producción foliar, contrario a lo que se esperaba.

Las tasas de recambio foliar son altas cuando hay mu-

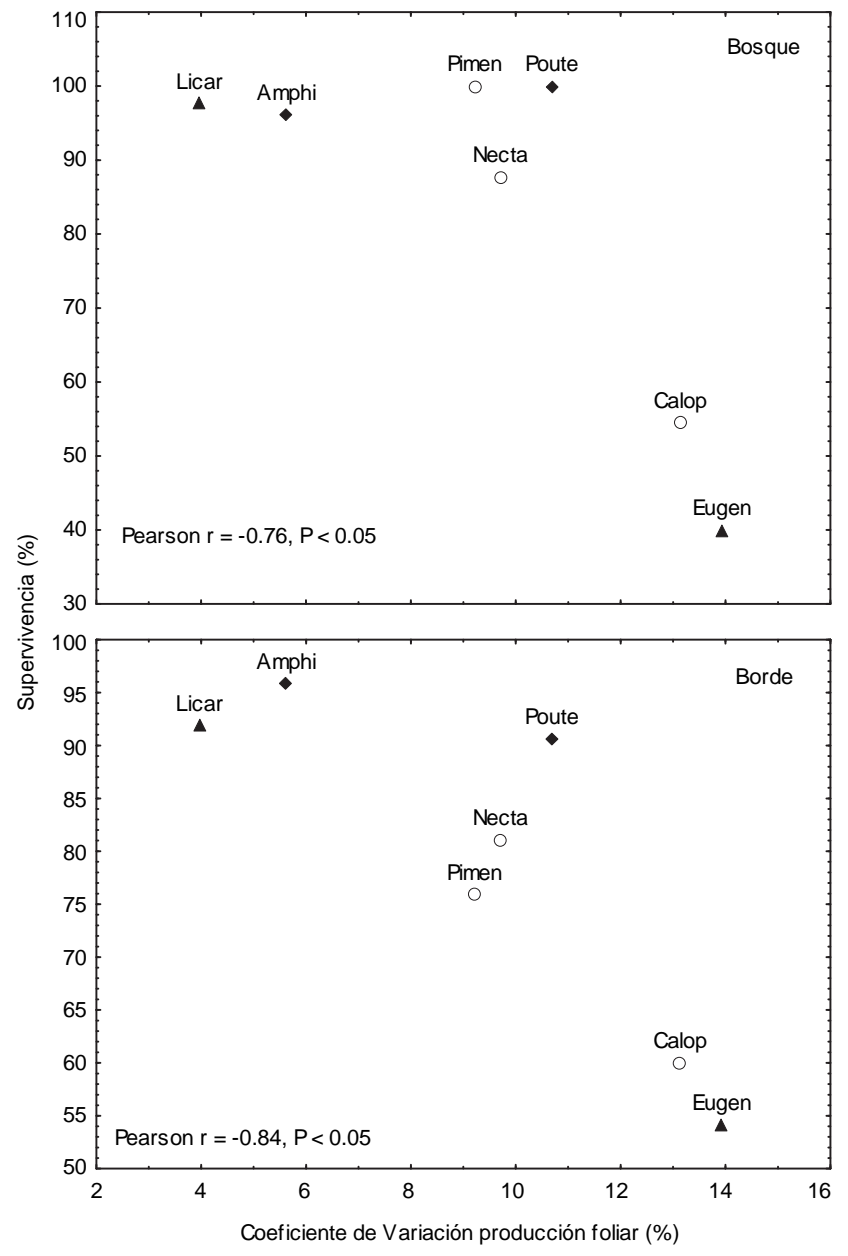

Figura 4. Correlación de la supervivencia de los individuos (\%) y la plasticidad de la producción foliar (Coeficiente de Variación en la producción foliar \%) de siete especies sucesionales tardías en bosque secundario y borde de Los Tuxtlas, Veracruz, Mexico. Los círculos corresponden a árboles del dosel, los triángulos al dosel medio y los diamantes a los árboles que habitan el sotobosque. Se muestran las primeras cinco letras del género para cada especie.

cha luz, como en los claros o en la parte superior del dosel, mientras que plantas creciendo en el sotobosque muestran tasas bajas de recambio foliar. Sólo un estudio ha medido la pérdida de hojas de juveniles de especies sucesionales tardías en áreas perturbadas (Reich et al., 2004), y se encontraron resultados similares: baja longevidad foliar comparada con los valores de juveniles y adultos del bosque. Esto no sorprende si se toma en cuenta que en el bosque tropical primario los niveles de luz a un metro del dosel (ca. 30-40 m de altura) pueden ser similares a aquellos medidos en áreas abiertas, como en deslaves, áreas incendiadas o pastizales (Richards, 1975). Lo que es novedoso es cómo estas diferencias en demografía foliar se relacionan con la supervivencia y el crecimiento de árboles sembrados dentro y fuera 
del bosque. En este estudio se encontró una variación baja en la supervivencia de hojas (1.1 veces) y en la producción foliar (2.5 veces) para las siete especies estudiadas, lo cual se espera para un grupo de especies que comparten la misma historia de vida. Sin embargo, aún esta variación baja en la demografía foliar, se correlacionó con la supervivencia y el crecimiento de juveniles en los diferentes hábitats.

La relación entre la supervivencia de las plantas y la supervivencia de las hojas es distinta para cada hábitat. Dentro del bosque tropical de Bolivia, la supervivencia de juveniles de 25 especies estuvo positivamente correlacionada con la longevidad de sus hojas (retención de hojas), lo cual reflejó una estrategia de conservación de recursos de individuos creciendo en la sombra (Poorter y Bongers, 2006). En este estudio, una menor supervivencia foliar estuvo asociada con una alta supervivencia de las plantas cuando fueron sembradas en el pastizal. Una menor supervivencia foliar puede reflejar un compromiso para disminuir el uso de agua; entonces, individuos que tiran sus hojas rápidamente en el pastizal tienen mayor supervivencia debido a que disminuyen su transpiración en condiciones de estrés hídrico (Holl, 1999). En este estudio, Nectandra, un árbol dominante del dosel del bosque de Los Tuxtlas, Licaria, un árbol del dosel medio, y Pouteria, un árbol pequeño del sotobosque, tuvieron las tasas más altas de supervivencia en el pastizal tirando sus hojas rápidamente. Especies capaces de tirar sus hojas en condiciones de alta luminosidad (áreas perturbadas) y de retenerlas en condiciones de sombra (sotobosque secundario o primario) tendrán mayores posibilidades de crecer y sobrevivir en sitios bajo perturbación, regeneración natural o sucesión dirigida (restauración).

Una producción foliar alta es ventajosa sólo en ciertas situaciones. En un estudio en el bosque de Los Tuxtlas, se encontró que las tasas de crecimiento de juveniles de ocho especies estuvieron positivamente correlacionadas con el promedio de producción foliar para individuos creciendo en claros, pero no para aquellos creciendo en el sotobosque (Bongers y Popma, 1990; King, 1994). Se ha visto que las especies que tienen una producción foliar alta, mueren si se encuentran en condiciones de bajos recursos; por ejemplo, en la selva tropical de Panamá se encontró que las pioneras germinaban en el sotobosque pero su producción foliar alta sólo podía ser mantenida por un corto periodo con la luz existente bajo el dosel (King, 1994) ocasionando un gasto total de energía (sensu Lusk, 2004). En este estudio no encontramos correlación de la supervivencia o del crecimiento de las plantas con la producción foliar. Dado que el intervalo de variación en la cantidad de luz dentro de cada hábitat es alta, la plasticidad en la demografía foliar puede predecir mejor el crecimiento y la supervivencia de los individuos que el promedio pues el coeficiente de variación refleja una respuesta plástica con mayor valor adaptativo.

La plasticidad en la demografía foliar nunca ha sido correlacionada con la supervivencia y el crecimiento de las plantas. Las especies sucesionales tardías del bosque tropical perennifolio enfrentan cambios azarosos en el ambiente lumínico ocasionados por caída de ramas y árboles, defoliación y crecimiento de árboles vecinos, lo que libera y suprime su crecimiento mediante adaptaciones fisiológicas y estructurales (Bongers y Popma, 1988; Canham, 1989). Es posible que una alta plasticidad en la retención y producción de hojas pueda favorecer el éxito de especies sucesionales tardías en hábitats que contrastan en cantidad de luz y agua, como el pastizal y el bosque. En este estudio, las especies con mayores tasas de supervivencia y crecimiento en el pastizal fueron aquellas con mayor plasticidad en la supervivencia foliar, por ejemplo, Nectandra y Licaria. Además, estas especies también presentaron una alta supervivencia en el borde y en el bosque, asociada a su plasticidad en la supervivencia foliar. Por otra parte, Amphitecna, un árbol pequeño del sotobosque tuvo una tasa alta de supervivencia sólo en el bosque y en el borde. Nuestros datos sugieren que plantas que tienen una respuesta inmediata a los diferentes niveles de luz, tirando sus hojas en las áreas expuestas y reteniéndolas en el bosque, tendrán las más altas tasas de crecimiento y supervivencia en los hábitats más comunes del paisaje mundial actual: los pastizales, los bordes y el bosque secundario.

Las especies que usualmente crecen en el sotobosque tienen características relacionadas con el "síndrome de resistencia al stress" (Chapin et al., 1993), es decir, tienen tasas bajas de crecimiento y de recambio de hojas aún cuando están expuestas a condiciones de alta disponibilidad de recursos (estabilidad fenotípica). Por ejemplo, la baja producción foliar es adaptativa en el sotobosque porque evita el traslape de hojas e incrementa la captura de luz (Miyazawa et al., 2006). En nuestro estudio, las siete especies sucesionales tardías incrementaron muy poco su producción foliar en el pastizal; la producción foliar y su variación para los tres hábitats fueron bajas, lo que sugiere que la plasticidad en la producción foliar no confiere ventaja a estos juveniles. Aparentemente, la estrategia de conservación de recursos de las especies evaluadas consistió en retener las hojas en el sotobosque, tirarlas en el pastizal y, producir pocas hojas incluso en áreas con mucha luz como el pastizal. La preadaptación más exitosa parece ser evitar la pérdida de agua y el gasto de energía hasta que los juveniles en los pastizales se encuentren bajo sombra para comenzar a crecer otra vez.

Las especies sucesionales tardías representan más del $80 \%$ de las especies del bosque primario y proveen de diversidad estructural y de alimento a los animales que lo habitan. En los lugares donde la regeneración se da de manera natural (i.e., cerca de fragmentos de bosque), las pioneras pueden llegar mediante eventos de dispersión y las sucesionales tardías deben ser sembradas para acelerar la recuperación de la biodiversidad (Martínez-Garza y Howe, 2003). En áreas abiertas, recientemente abandonadas o excluidas de la perturbación se recomienda sembrar una mezcla de 
pioneras que detengan la erosión y de sucesionales tardías que aumenten la biodiversidad. Basados en nuestros resultados, se recomienda sembrar Nectandra, Licaria y Pouteria en pastizales de Los Tuxtlas ya que tuvieron tasas altas de crecimiento y supervivencia en ese hábitat; además, estas especies proveerían de diversidad estructural al bosque resultante, ya que alcanzan diferentes estaturas cuando adultas. Especies con baja supervivencia en pastizales como Calophyllum, Pimenta, Amphitecna y Eugenia podrían sembrarse en grandes cantidades o hasta que un dosel de pioneras se haya formado. Los proyectos de restauración ecológica siempre deberían incluir tantas especies como sea posible para maximizar la diversidad y complejidad de los bosques restaurados.

\section{Agradecimientos}

Agradecemos al personal de la Estación de Los Tuxtlas, a Jose Luis Paxtian por su ayuda en el campo y a Alejandro Flores-Palacios y dos revisores anónimos por sus comentarios al manuscrito. C. M-G fue apoyada por CONACYT (beca de Doctorado No. 115974), Lincoln Park Zoo de Chicago, y la NSF de Estados Unidos (DEB No. 0516259 para H.F.H.) en la etapa de escritura.

\section{Literatura Citada}

Allen E.B., Covington W.W. y Falk D.A. 1997. Developing a conceptual basis for restoration ecology. Restoration Ecology 5:275-276.

Begon, M., Harper, J.L. y Townsend, C.R. 1987. Ecología: Individuos, Poblaciones y Comunidades. Ediciones Omega, Barcelona.

Benitez-Malvido J., Martínez-Ramos M., Camargo J.L.C. y Ferraz I.D.K. 2005. Responses of seedling transplants to environmental variations in contrasting habitats of Central Amazonia. Journal of Tropical Ecology 21:397-406.

Bongers F. y Popma J. 1988. Is exposure-related variation in leaf characteristic of tropical rain forest adaptive? En: Werger M.J.A., Van-der-Aart P.J.M., During H. J. y Verhoeven, J.T.A. eds. Plant Form and Vegetation Structure pp.192-200. Academic Publishing, Holanda.

Bongers F., y Popma J. 1990. Leaf dynamics of seedlings of rain forest species in relation to canopy gaps. Oecologia 82:122127.

Bongers F., Popma J., Meave-del-Castillo J., y Carabias J. 1988. Structure and floristic composition on the lowland rain forest of Los Tuxtlas, Mexico. Vegetatio 74:55-80.

Bradshaw A.D. 1965. Evolutionary significance of phenotypic plasticity in plants. Advances in Genetics 13:115-155.

Canham C.D. 1989. Different responses to gaps among shade-tolerant tree species. Ecology 70:548-550.

Carabias-Lillo J. y Guevara-Sada S. 1985. Fenología de una selva tropical húmeda y en una comunidad derivada; Los Tuxtlas, Veracruz. En: Gómez-Pompa A. y del-Amo S. eds. Investigaciones Sobre Regeneración de Selvas Altas en Veracruz, México pp. 27-78. Alhambra Mexicana, México D.F.
Clark D.A. y Clark D.B. 1992. Life-history diversity of canopy and emergent trees in a Neotropical rain forest. Ecological Monographs 62:315-344.

Coley P.D. 1988. Effects of plant-growth rate and leaf lifetime on the amount and type of anti-herbivore defense. Oecologia 74:531-536.

Chabot B.F. y Hicks D.J. 1982. The ecology of leaf life spans. Annual Review of Ecology and Systematics 13:229-259.

Chapin F.S., Autumn K. y Pugnaire F. 1993. Evolution of suites of traits in response to environmental stress. American Naturalist 142:S78-S92.

Chapman, S.B. 1976. Methods in Plant Ecology. Blackwell Scie Pub, Oxford.

Holl K.D. 1999. Factors limiting tropical rain forest regeneration in abandoned pasture: seed rain, seed germination, microclimate, and soil. Biotropica 31:229-242.

Holl K.D. y Howarth R.B. 2000. Paying for restoration. Restoration Ecology 8:260-267.

Ibarra-Manriquez G. y Sinaca, S. 1995. Lista florística comentada de la Estación de Biología Tropical "Los Tuxtlas", Veracruz, México. Revista de Biología Tropical 43:75-115.

Ibarra-Manriquez G. y Sinaca S. 1996a. Lista florística comentada de la Estación de Biología Tropical "Los Tuxtlas", Veracruz, México (Mimosaceae a Verbenaceae). Revista de Biología Tropical 44:41-60.

Ibarra-Manriquez G., y Sinaca S. 1996b. Lista florística comentada de la Estación de Biología Tropical "Los Tuxtlas", Veracruz, México (Violaceae a Zingiberaceae). Revista de Biología Tropical 44:427-447.

Jordan W.R., Gilpin M.E. y Aber J.D. 1987. Restoration ecology: ecological restoration as a technique for basic research. En: Jordan W.R., Gilpin M.E. y Aber J.D. eds. Restoration Ecology: A Synthetic Approach to Ecological Research pp.3-22 Cambridge University Press, Cambridge.

King D.A. 1994. Influence of light on the growth and morphology of saplings in a Panamanian forest. American Journal of Botany 81:948-957.

Lambers H. y Poorter H. 1992. Inherent variation in growth rate between higher plants: A search for physiological causes and ecological consequences. En: Begon M. y Fitter A.H. eds. Advances in Ecological Research Vol. 23 pp.187-261. Academic Press, London.

Lortie C.J. y Aarssen L.W. 1996. The specialization hypothesis for phenotypic plasticity in plants. International Journal of Plant Sciences 157:484-487.

Lusk C.H. 2004. Leaf area and growth of juvenile temperate evergreens in low light: species of contrasting shade tolerance change rank during ontogeny. Functional Ecology 18:820-828.

Martin-Del-Pozzo A.L. 1997. Geología. En: Gonzélez-Soriano E., Dirzo R. y Vogt R.C. eds. Historia Natural de Los Tuxtlas pp.25-31. UNAM-CONABIO, Mexico, D.F.

Martínez-Garza C. y González-Montagut R. 1999. Seed rain from forest fragments into tropical pastures in Los Tuxtlas, Mexico. Plant Ecology 145:255-265.

Martínez-Garza C. y Howe H.F. 2003. Restoring tropical diversity: beating the time tax on species loss. Journal of Applied Ecology 40:423-429.

Martinez-Garza C. y Howe H.F. 2005. Developmental strategy or immediate responses in leaf traits of tropical tree species? International Journal of Plant Sciences 166:41-48. 
Martínez-Garza C., Pena V., Ricker M., Campos A. y Howe H.F. 2005. Restoring tropical biodiversity: Leaf traits predict growth and survival of late-successional trees in early-successional environments. Forest Ecology and Management 217:365-379.

Meave-del-Castillo J.A. 1987. Longevidad de las hojas de tres especies de árboles perennifolios de la selva tropical húmeda. Tesis de Maestría, Facultad de Ciencias, Universidad Nacional Autónoma de México, México, D.F. 96 pp.

Miyazawa Y., Ishihara M., Suzuki M., Fukumasu H. y Kikuzawa K. 2006. Comparison of the physiology, morphology, and leaf demography of tropical saplings with different crown shapes. Journal of Plant Research 119:459-467.

Moran E.F., Brondizio E., Mausel P. y Wu Y. 1994. Integrating Amazonian Vegetation, Land-Use, and Satellite Data. Bioscience 44:329-338.

Osada N., Takeda H., Furukawa A. y Awang M. 2001. Leaf dynamics and maintenance of tree crowns in a Malaysian rain forest stand. Journal of Ecology 89:774-782.

Poorter L. y Bongers F. 2006. Leaf traits are good predictors of plant performance across 53 rain forest species. Ecology 87:1733-1743.

Popma J., Bongers F. y Werger M.J.A. 1992. Gap-dependence and leaf characteristics of trees in a tropical lowland rain forest in México. Oikos 63:207-214.

Reich P.B., Uhl C., Walters M.B. y Ellsworth D.S. 1991. Leaf lifespan as a determinant of leaf structure and function among 23 Amazonian tree species. Oecologia 86:16-24.

Reich, P.B., Walters, M.B., y Ellsworth, D.S. 1992. Leaf life-span in relation to leaf, plant, and stand characteristics among diverse ecosystems. Ecological Monographs 62:365-392.

Reich P.B., Uhl C., Walters M.B., Prugh L. y Ellsworth D.S. 2004. Leaf demography and phenology in Amazonian rain forest: A census of 40000 leaves of 23 tree species. Ecological Monographs 74:3-23.

Reich P.B., Wright I.J., Cavender-Bares J., Craine J.M., Oleksyn J., Westoby M. y Walters M.B. 2003. The evolution of plant functional variation: Traits, spectra, and strategies. International Journal of Plant Sciences 164:S143-S164.

Richards P.W. 1975 The Tropical Rain Forest: An Ecological Stu$d y$. Cambridge University Press, London.

Schlichting C.D. y Levin D.A. 1984. Phenotypic Plasticity of Annual Phlox: Tests of Some Hypotheses. American Journal of Botany 71:252-260.

Siegel S. y Castellan N.J. 2005. Estadística No-Paramétrica: Aplicada a las Ciencias de la Conducta. Trillas, Mexico, D.F.

StatSoft Inc. 2004. STATISTICA for Windows. Manual version 7.0. StatSoft Inc. Tulsa, Oklahoma.

Valladares F., Wright S.J., Lasso E., Kitajima K. y Pearcy R.W. 2000. Plastic phenotypic response to light of 16 congeneric shrubs from a Panamanian rainforest. Ecology 81:1925-1936.

Wijdeven S.M.J. y Kuzee M.E. 2000. Seed availability as a limiting factor in forest recovery processes in Costa Rica. Restoration Ecology 8:414-424.

Zimmerman J.K., Pascarella J.B. y Aide T.M. 2000. Barriers to forest regeneration in an abandoned pasture in Puerto Rico. Restoration Ecology 8:350-360.

Recibido: 3 de marzo del 2009

Aceptado: 17 de noviembre del 2009 